\title{
VELOCITY PROFILE GENERATION FOR TRAJECTORIES OF A SELF-DRIVING CAR
}

\section{HRISHIKESH DEY \& RITHIKA RANADIVE}

Department of Electronics Engineering, VES Institute of Technology, Mumbai, India

\begin{tabular}{|l|}
\hline ABSTRACT \\
Velocity planning is one of the core functionalities of an autonomous driving system. This involves determining velocity \\
at every instant of time for smooth and continuous motion of the autonomous system along a given path. In this paper an \\
analytical method based on most employed trapezoidal velocity profile is proposed for generating velocities for a vehicle \\
traveling along a predefined path. To improve comfort and to provide smooth control, s-like trapezoidal profile is \\
considered employing cubic spline for the ramp-up and ramp-down portions. Depending upon various acceleration and \\
velocity constraints which are derived from the road and physical systems, the trapezoidal velocity profile is either a ramp- \\
up or ramp-down or ramp-up-constant-velocity or constant-velocity-ramp-down or trapezoidal profile, which is suitably \\
associated with the given path. An in-depth analysis of the proposed method along with constraints comes from practical \\
driving consideration and the results are presented in this paper. \\
KEYWORDS: Autonomous Vehicles, Motion Planning, Trapezoidal Velocity Profile, Polynomial Splines
\end{tabular}

Received: Jun 06 2021; Accepted: Jun 26, 2021; Published: Jul 14, 2021; Paper Id.: IJRRDDEC20211

\section{INTRODUCTION}

Autonomous driving has been predicted to dramatically enhance driving safety, increase transportation efficiency and revolutionize the entire automobile industry, where particularly self-driving cars can offer tremendous benefits to both individuals and societies [1]. With the help of the on-board technologies, self-driving cars can perform realtime driving tasks without any input from a human operator by creating an image of the surroundings to facilitate traffic navigation [1, 2]. Apart from basic navigation, self-driving vehicles offer increased road safety, and may unclog road traffic due to their ability to communicate with each other, thereby optimizing vehicular routes and thus, resulting in smooth and efficient traffic flow. This, in turn, results in decreased fuel consumption, hence, paving the way for a more sustainable future [3,4]. For the plethora of advantages these vehicles offer, they have been subjected to intense research, and thus, have become a definite reality that may pave the way for future systems, where robots take over the art of driving [3, 4].

To achieve human-like capabilities while driving, autonomous driving cars rely on path planning, this is the "brain" of the entire system. Path planning and decision making in urban environments enable self-driving cars to discover the safest, most convenient, and most economical routes from source to destination [5, 6]. This subsystem is capable of reproducing the human thought-process involved while driving - route planning based on the source and destination, real-time analysis of the surroundings and maneuvering any encountered obstacles, and at the same time adhering to traffic rules and maintaining a safe and optimal trajectory [7, 8]. As autonomous on-road driving require path, manoeuvre and trajectory planning, the planning algorithm includes the search for an optimal collision-free path adhering to the feasible configurations implicated by path planning, characterization of motion 
indicated by manoeuvre planning, and real-time constraints while transitioning from one feasible state to another as directed by trajectory planning $[9-11]$.

The path planning algorithm includes the search for a feasible path by taking into consideration the dimensions of the vehicle, the geometry of its surroundings, and the various kinematical path constraints $[9,12]$. The vehicle's movement is calculated so as to fulfill the car's kinematic constraints based on its initial and final dynamic configurations. The vehicle must identify and bypass all the static and maneuverable obstacles to find a collision-free route. Following the path planner's generation of a route through the road network, the man oeuvre/motion planner evaluates the surrounding environment and produces the most appropriate motion specification for the trip. The most suitable path is obtained using a trajectory planning algorithm which refers to the creation of a collision free path from the initial point to the destination point. The objective of trajectory planning is to search for the optimal motion of a vehicle subject to obstacle fields and vehicle motion constraints. In order to generate the real-time trajectories, a reference path (base frame) is extracted mathematically considering various path constraints such as restrictions on size of vehicle with respect to the road, and restrictions on vehicular drive. Using this base frame as a reference, a rich set of possible paths (candidate frames) for a given source and destination will be generated kinematically. Each path generated is then associated with one or many velocity profiles. The most feasible candidate is then selected based on the criteria of collision avoidance with both static and dynamic obstacles, vehicle constraints and smooth driving. Motion planning highlights various critical challenges in the development of the autonomous driving systems, including velocity planning. Velocity planning, defined as the variation in velocity along a predefined path for the motion control of autonomous vehicles, becomes very complex due to continuously changing environments $[13,14]$. Therefore, the velocity profile needs to be carefully assigned on each point along the generated path. By considering both path planning and motion planning constraints, a velocity profile is employed that can optimize driving capability, while adhering to the behavior and velocity limits of the vehicles in a driving scenario, and at the same time avoids collision completely [15].

In recent years, a significant amount of work has been dedicated to achieve fast and efficient motion planning algorithms, as it remains one of the most important aspects of autonomous driving. Aurelio Piazzi in [2] proposed a solution that includes designing a longitudinal velocity profile incorporating cubic splines and an ad hoc algorithm with a minimum jerk, eventually accomplishing the creation of continuous velocities and accelerations. As demonstrated by Xiaohui $\mathrm{Li}$ in [16], the velocity profile generation is initiated by the determination of maximum permitted speed by the behavioural planner, the model constructs the trapezoidal speed curve, followed by the application of polynomial splines to ensure continuous acceleration. The formal proof to produce an optimal velocity profile, for least time travel, has been suggested in [17] by overcoming the loss of controllability that occurred in previous literature providing faster computation along with the ease of implementation. Keonyup Chu in [1] described a collision risk procedure to determine target speed by limiting the value of lateral acceleration as maintained by the curvature of the path. Jordi P'erezTalamino and Alberto Sanfeliu's method in [13] imposed initial and final acceleration restrictions to compute the profiles using third order velocity splines in four unknown variables, considering the fifth unknown to be the total time required to travel the given trajectory. The latest development by I. Bae in [18] planned an Occupant's Preference Metric (OPM) which described a lateral and longitudinal acceleration section with the highest permissible jerk.

Autonomous vehicles should be capable of assigning appropriate velocity profiles for a selected optimal path. Velocity planning is a versatile and effective tool for the motion control of autonomous vehicles. The planning 
optimization algorithm should offer an efficient method for the evaluation of minimum pure-jerk velocity functions. To ensure smooth driving, a velocity planner uses semantic information given by the path planner to establish a continuous velocity profile that considers acceleration and speed thresholds within the selected optimal path (trajectory) [19]. This paper provides an overview to achieve generation of suitable velocity profiles for an optimal trajectory, explicitly considering various velocity, acceleration, path and time constraints. Considering these constraints help reduce the solution set of the velocity planning, resulting in the planner to focus on the solution space where the optimal solution is more likely to exist. The trapezoidal velocity profile is considered as a reference to generate a stable velocity profile fulfilling various constraints and mode of travel such as velocity-keeping, following, stopping etc. as suggested by higher order behavioral module. Finally, to obtain smooth and jerk free motion the generated velocity profile is replaced with high order polynomial spline curve maintaining all constraints.

Specifically, the main contributions of this paper are:

- An approach for accurately generating suitable velocity profiles by employing trapezoidal velocity curve for anoptimal trajectory, explicitly considering various velocity, acceleration, path and time constraints.

- Demonstration of different jerk minimization techniques involving generation of smooth s-curve motion profiles with low associated accelerations, incorporating high order polynomial spline equations with the suitably generated trapezoidal velocity profile sequences for the optimal trajectory.

- An experimental validation and evaluation of the special test case where velocity and time constraints are altered to generate the suitable motion profile.

\section{RELATED WORK}

Over the last decade, there are ongoing efforts to deploy driver-less cars in realistic urban scenarios. This section deals with the assessment of various complex techniques that have been produced for the accurate generation of optimal trajectories and implementation of motion profiles for the development of autonomous vehicles. Previous research has attempted to reduce jerks in autonomous vehicles by applying various smooth velocity profiles, such as the s-curve jerk-bounded profile. High-order polynomial motion profiles enable the vehicle to move smoothly. The increase in the order of a polynomial equation results in an equivalent increase in the number of coefficients for the function of time, resulting in the generation of smoother shapes of position and velocity profiles. Abhiram Rahatgaonkar's thesis on Velocity Planning Approach for Autonomous Vehicles [20] concentrates on velocity planning with familiar environment to avoid collision with moving obstacles. This technique involves time scheduling in space along with $\mathrm{A}^{*}$ search algorithm to optimize the generated profile. The specified analysis promises to extend towards greater driving scenarios including merging and lane intersection. Further research in [21] asserted a practice involving the calculation of minimum velocity for the entire trajectory to construct a smooth transition profile, from the minimum to the maximum allowed speed, fulfilling acceleration and jerk restraints. Robust Motion Predictive Control (RMPC) design methodology presents a system capable of handling both ordinary and rare driving scenarios. This approach recommends considering the velocity and acceleration constraints of the preceding vehicle as instantaneous checks instead of constant values for the entire trajectory to be followed. It calculates the relative velocity and distance at every time instant for generating acceleration sequence adhering to the collision avoidance ability. The safety constraints applied to the vehicle are actuator limit, speed limit and safe 
following distance. Thus, Francesco Borrelli in [22] achieved significant accuracy and safety control through his proposed framework.

Zvi Shiller in [23] achieves a path planning algorithm for vehicles moving on a general terrain and accounts for obstacles, terrain topography, vehicle kinematics and dynamics. The terrain and the path are illustrated by B spline patches and curves. This method determines the most feasible path requiring minimum time and adhering to vehicle kinematics, the engine torque limit and the given coefficient of friction. The mathematical derivation of the desired torque or velocity signals that act as an input to the respective torque or speed controllers is provided by Martin Adams in [24]. In addition to that, it also defined a trajectory generator which produced constant jerk profiles in acceleration, velocity and displacement, while maintaining vehicular constraints of acceleration and velocity. Seiji Sugiyama in [25] proposed a method by applying an ellipse field, which is obtained by using the conformal transformation, and a correction function, which generates the continuous velocity field, to an already existing Hydrodynamic Potential method for path planning of a mobile robot. The system when applied to arbitrarily moving obstacles, showed results with reduced velocity variation. Cheng Chen in [26] devised a solution to counter the problem of trajectory generation by producing continuous and bounded curvature profile to outline the trajectory, further optimized by the quartic B'ezier curve. The structure of linear velocity profile generation has also been proposed to avoid sideslip, and establish velocity-continuity and acceleration limits. An algorithm of computational complexity $\mathrm{O}(\mathrm{n})$ has been used by L. Consolini in [27] to provide a time-optimal velocity planning technique for autonomous vehicles. Not only does this planning take into account the usual kinematic constraints, but also sets a bound on the vehicle's maximum normal acceleration which aids in avoiding dangerous slips.

\section{VELOCITY PLANNING FORMULATION}

We designed and implemented an efficient real-time approach for velocity planning of a known optimal trajectory within specified constraints as shown in Algorithm 1. The required velocity and acceleration constraints can be calculated/modified such that the trapezoidal velocity profile modifies to a linear or a ramp velocity profile.
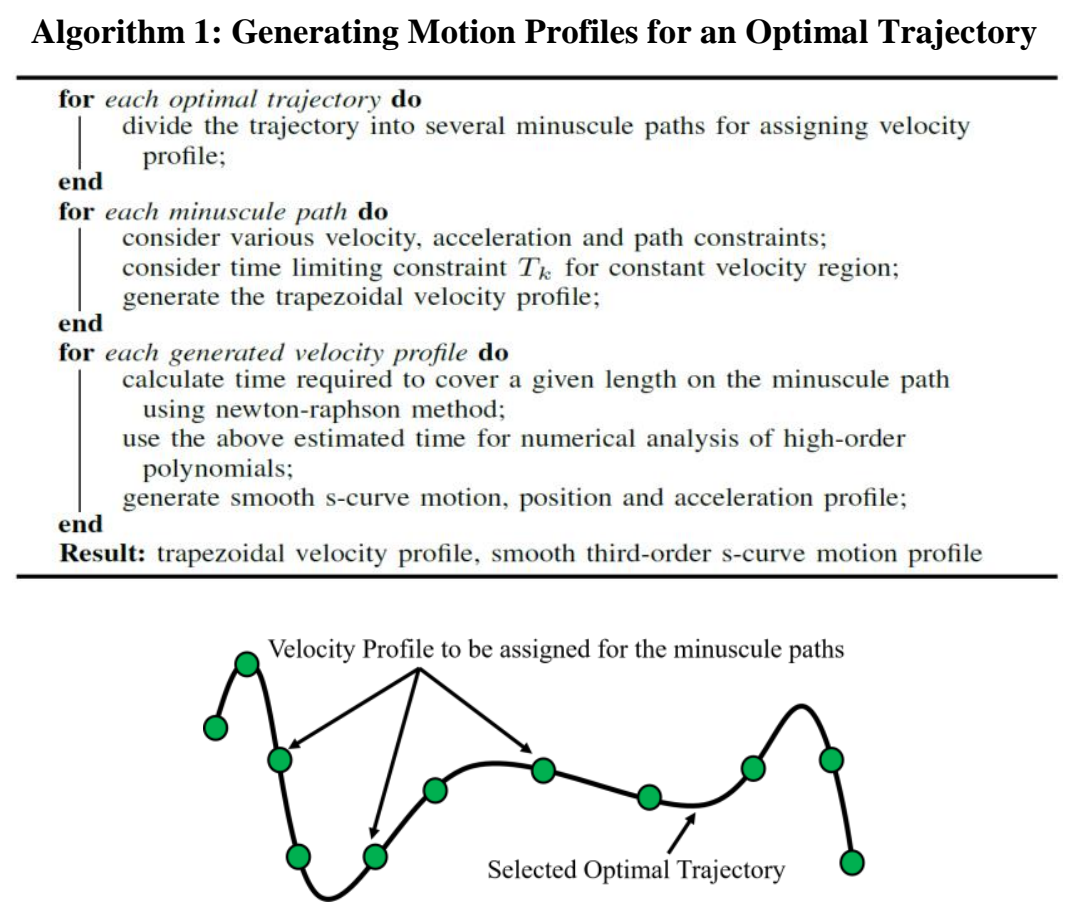

Figure 1: The Division of Selected Optimal Trajectory into Minuscule Paths for Assigning Velocity Profile 
Assumptions. Considering the velocity (initial velocity $V_{o}$, maximum velocity $V_{m}$ and end velocity $V_{e}$ ) and acceleration (initial acceleration $A$ and deceleration $D$ ) constraints, a velocity profile can be designed for a selected optimal trajectory(Total path length $S$ ).

Algorithm Phase 1. The initial procedure involves the generation of a speed curve by employing the trapezoidal velocity profile. Instead of generating the velocity profile for the entire path length $S$, we divide the trajectory into several minuscule paths of length $s$ to obtain the best fitting curve as illustrated in Figure 1. The instantaneous changes in velocity demand the constraints to differ for every minuscule path length $s$.

Algorithm Phase 2. The initial velocity $V_{o}$ for the first segment is established in advance by the planner. The maximum velocity $V_{m}$ remains constant for the given minuscule path and restricts the vehicle from exceeding the range. We introducea new constraint, the limiting constant, $T_{k}$, which is the least time for which the vehicle is expected to travel at a constant maximum velocity to avoid collisions and maintain stability. The final velocity $V_{e}$ is dynamically dependent on the velocity of the leading vehicle to ensure collision free driving. As shown in Figure 2, the trapezoidal motion profile is applied for the velocity profile generation. We assume the ramp-up and ramp-down profiles with constant acceleration and deceleration values to attain a solution. The velocity profile consists of three sections: (1) the initial ramp-up slope $T_{l} / S_{l}$ from the initial velocity $V_{o}$ till the maximum velocity $V_{m} ;(2)$ constant traversal segment $T_{2} / S_{2}$ at the maximum velocity $V_{m}$; (3) the ramp-down slope $T_{3} / S_{3}$ from the maximum velocity $V_{m}$ till the final velocity $V_{e}$. The equations for distance covered by the three sections $S_{1}, S_{2}$ and $S_{3}$ have been formulated using Newton's Laws of Motion. The consecutive minuscule path segments obtain the final velocity $V_{e}$ of the previous set and set it as its initial velocity $V_{o}$.

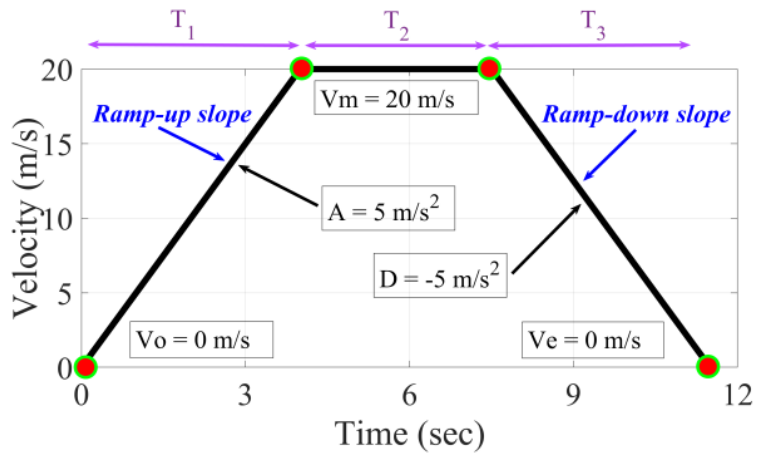

Velocity v/s Time

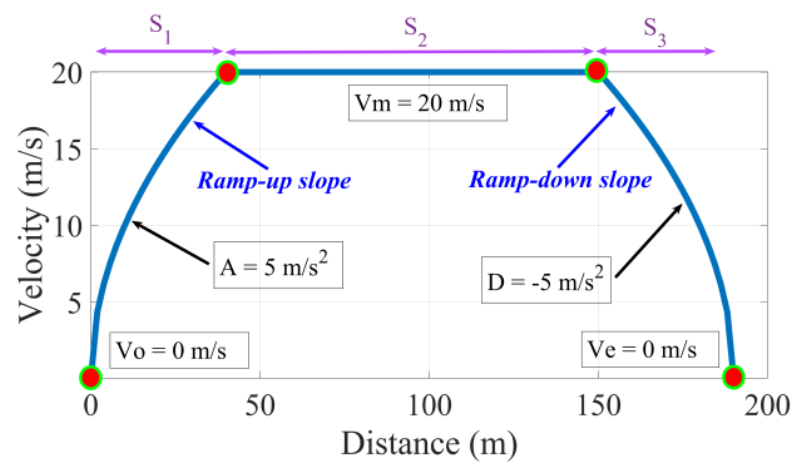

Velocity v/s Distance

Figure 2: Trapezoidal Velocity Profiles Showing Different Segments. 


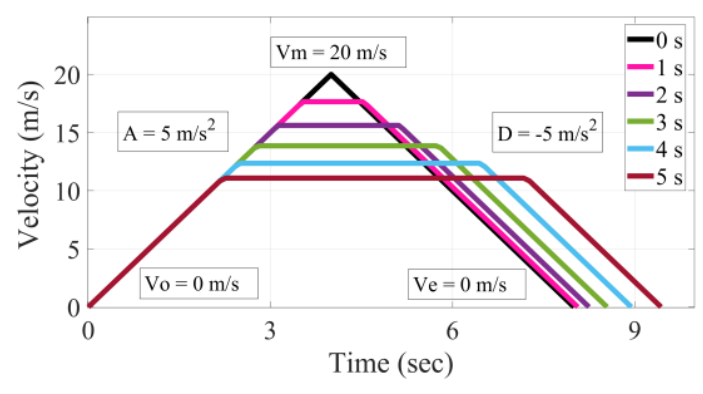

Figure 3: Trapezoidal Velocity Profile Considering $T_{k}$ to vary from 0 to 5 seconds.

$$
\begin{aligned}
& S_{1}=\frac{V m^{2}-V o^{2}}{2 \times A} \\
& S_{2}=V_{m} \times t_{s 2} \\
& S_{3}=\frac{V e^{2}-V m^{2}}{2 \times D} \\
& s=S_{1}+S_{2}+S_{3} \\
& t_{S 2}=\frac{s-\left(S_{1}+S_{3}\right)}{V_{m}} \\
& n e w V_{m}=\frac{-T_{k}+\sqrt{T_{k}{ }^{2}-4 \times\left(\left(\frac{1}{2 \times A}\right)-\left(\frac{1}{2 \times D}\right)\right) \times\left(\left(\frac{V e^{2}}{2 \times D}\right)-\left(\frac{V o^{2}}{2 \times A}\right)-s\right)}}{2 \times\left(\left(\frac{1}{2 \times A}\right)-\left(\frac{1}{2 \times D}\right)\right)}
\end{aligned}
$$

The total distance of the minuscule path $s$ in Equation 4 for which the velocity profile has been generated, is equal to the sum of the distance covered by three segments of the profile as specified in Equations 1, 2 and 3. Considering the total path length to remain constant, and acceleration and deceleration to be $A$ and $D$ respectively, the time $t_{S 2}$ for traveling the segment $S_{2}$ is estimated in Equation 5.

The velocity profile generated in Figure 3 is a special case as it does not trace a zero-acceleration region when $T_{k}$ $=0$. The abrupt change from acceleration to deceleration results in a jerky motion. Thus, to ensure a smooth motion, a new value of maximum velocity, labeled new $V_{m}$, replaces $V_{m}$ while plotting the velocity curve. For this purpose, we have defined a constant $T_{k}$, which serves as a time limiting constant and fulfils the need to generate a non-jerky and smooth velocity profile for all sets of inputs. The constant, $T_{k}$, is the least time for which the vehicle is expected to travel at a constant velocity $V_{m}$ to avoid collisions and maintain stability. If the calculated time $t_{S 2}$ in Equation 5 is greater than or equal to $T_{k}$, there is no change in the value of $V_{m}$ as the time stability criterion is already obeyed. However, in cases where the estimated time $t_{S 2}$ turns out to be lesser than $T_{k}$, new $V_{m}$ is calculated using a quadratic formula as mentioned in Equation 6. The equation for new $V_{m}$ is formulated by utilizing Equation 4. The time required for covering the segment $S_{2}$ is considered as $T_{k}$ and the entire equation is reconstructed to form a quadratic equation with the variable $V_{m}$. This is achieved by allowing the vehicle to travel the same distance in increased time duration for a stable journey. The value of new $V_{m}$ is estimated by considering the minuscule path length $s$ to be constant and the time $t_{S 2}$ in Equation 5 to be time limiting constant, $T_{k}$.

On comparing Equation 6 to the standard quadratic equation $a x^{2}+b x+c$, thereby fulfilling the condition $b^{2}-4 a c \geq$ 0 , obtains two roots as the value of new $V_{m}$. Between the roots obtained, the greater positive value of the two is selected as 
the new $V_{m}$. Depending on the values of the constraints, the velocity profile curve chooses to fit $\left(S_{l}, S_{2}, S_{3}\right) /\left(T_{1}\right.$, $T_{2}, T_{3}$ )individually, or a combination of these three segments. On the contrary, if the condition $b^{2}-4 a c$ becomes negative, no solution is obtained for the given set of the velocity profile. Figure 3 shows that the value of $T_{k}$ when set to zero, results in no alteration in the value of $V_{m}$ as time $t_{S 2}$ evaluated for segment $S_{2}$ is equal or greater than the value of $T_{k}$. However, when $T_{k}$ is set to a higher value, then the value of the maximum velocity $V_{m}$ alters itself to a lower value of new $V_{m}$ to adhere to the vehicle constraints. As a result, anon-smooth trapezoidal velocity profile curve is established, limited by the vehicle and path constraints.

Algorithm Phase 3. The trapezoidal velocity profile is further smoothened to guarantee the continuity of the acceleration and optimize comfort by providing minimum jerk. Parameterization of the velocity profile is achieved by interpolating cubic polynomials. The ramp-up and ramp-down processes of the trapezoidal velocity profile are smoothened individually using cubic order spline equations, considering the most suitable runtime during computation.

$$
\begin{aligned}
& v(t)=v_{0}+a t+b t^{2}+c t^{3} \\
& a c c(0)=a=a_{1} \\
& v\left(t_{f}\right)=v_{0}+a_{1} t_{f}+a_{2} t_{f}^{2}+a_{3} t_{f}^{3}=v_{f} \\
& a c c\left(t_{f}\right)=a_{1}+2 a_{2} t_{f}+3 a_{3} t_{f}^{2}=a_{f} \\
& s\left(t_{f}\right)=v_{0} t_{f}+\frac{a_{1} t f^{2}}{2}+\frac{a_{2} t f^{3}}{3}+\frac{a_{3} t f^{4}}{4}=s_{f} \\
& \left(\frac{a_{0}-a_{f}}{12}\right) t_{f}^{2}+\left(\frac{v_{f}+v_{0}}{2}\right) t_{f--S_{f}}=0 \\
& a_{2}=3\left(\frac{v_{f}-v_{0}}{t f^{2}}\right)-\left(\frac{2 a_{0}+a_{f}}{t_{f}}\right) \\
& a_{3}=\left(\frac{a_{f}-a_{0}}{3 t f^{2}}\right)-\left(\frac{2 a_{2}}{3 t_{f}}\right)
\end{aligned}
$$

The cubic expression for velocity $v(t)$ at any given time $t$ is defined in Equation 7. The time taken to cover the ramp-up and ramp-down segments will be different in the s-curve profile as compared to the trapezoidal velocity curve. To smoothen these segments in the s-curve, the total time $t_{f}$ equired by each segment is calculated. The expression for velocity at $t_{f}$, can be derived using Equation 7 and it is expressed in Equation 9. The equation for acceleration at $t_{f}$, i.e. $a c c\left(t_{f}\right)$ is derived in Equation 10, using the first derivative of the velocity expression Equation 9. The equation for path length at $t_{f}$, i.e. $s\left(t_{f}\right)$ is derived in Equation 11, by integrating the velocity expression Equation 9. Given the initial velocity $V_{o}$ and initial acceleration $a_{o}$, the unknown parameters $\left\{a_{1}, a_{2}, a_{3}, t_{f}\right\}$ in Equation 11 could be analytically solved with the help of Equations 7, 8, 9, 10 and 11.

The equation for $t_{f}$ is obtained in Equation 12, where $v_{o}$ is the initial velocity, $v_{f}$ is the terminal speed, $a_{0}$ is the initial acceleration and $a_{f}$ is the terminal acceleration. From Equation 12, two values of $t_{f}$ are obtained. The most suitable value among the two is obtained and $t_{f}$ is selected separately for ramp-up and ramp-down segments. These segments are smoothened separately for all values of $t$ from $0-t_{f}$ in the velocity v/s time s-curve profile. Similarly, $s_{f}$ is calculated separately for the two segments using Equation 11. After the value for $t_{f}$ has been determined, $v(t), a c c(t)$ and $s(t)$ have to 


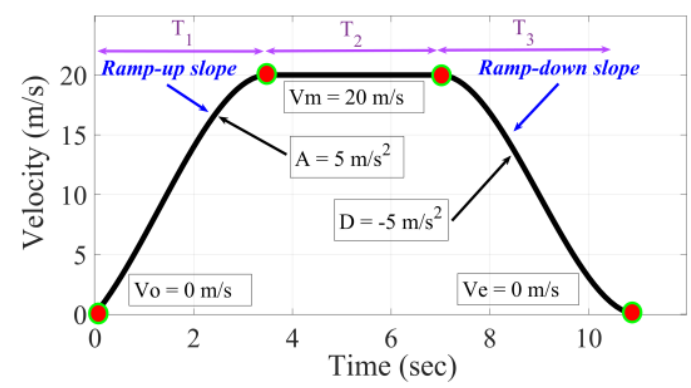

Velocity v/s Time

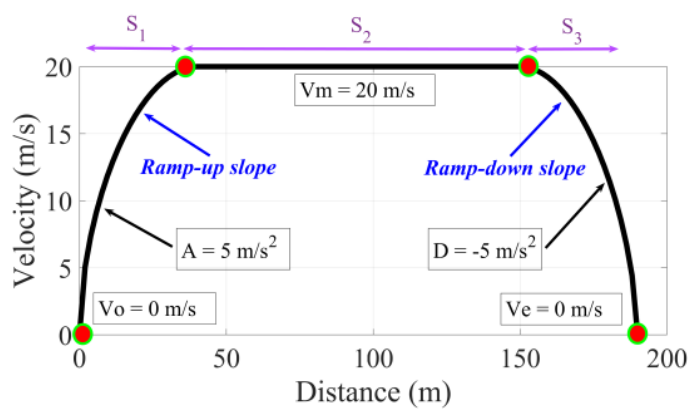

Velocity v/s Distance

Figure 4: Cubic s-Curve Velocity Profiles Showing Different Segments

be calculated in order to obtain the respective profiles. The equation for unknown parameters $a_{2}$ and $a_{3}$ can be methodically solved using Equations 7, 8, 9, 10 and 11, and are obtained in Equation 13 and 14, respectively. After the unknown parameters $\left\{a_{1}, a_{2}, a_{3}, t_{f}\right\}$ have been calculated and $t_{f}$ has been determined separately for ramp-up and ramp-down segments, $v(t), a c c(t)$ and $s(t)$ can be calculated for all values from $0-t_{f}$ using Equations 9, 10 and 11. With the help of Equations 9,10 and 11, the velocity, acceleration and distance s-curve profiles (plots) are obtained for all values from 0 - $t_{f}$ and thus, the profiles obtained are smoothened with the help of polynomial equations.

Newton-R aphson method, a root-finding algorithm that produces successively better approximations to the roots (or zeroes) of a real-valued function is used for calculating the value of $t_{f}$. The standard version of this method starts with a single-variable function $f\left[s\left(t_{f}\right)\right]$ defined for a real variable $t_{f}$, the function's derivative $f$ ', and an initial guess for a root of $f$. The value for the initial guess to is calculated by a pre-defined function which when given an input $s$, returns the time needed to cover $s$ distance. On considering that the function is able to satisfy necessary presumptions and the initial guess is very close, then the process mentioned below is repeated until an adequately accurate value is attained.

$$
t_{n+1}=t_{n}+\left(\frac{f\left(t_{n}\right)}{f^{\prime}\left(t_{n}\right)}\right)
$$

The value of $t_{f}$ is evaluated at every point on the trajectory, which is further employed to generate accelerationcontinuous velocity profiles. As shown in Figure 4, the S-shaped ramp-up and ramp-down velocity profiles are solved individually. Conclusively, trajectories are generated by incorporating the spatial path with the corresponding velocity profiles. 


\section{RESULTS AND DISCUSSION}

\section{A. Experimental Setup}

For simulating results and experimental evaluation, the entire programming code was developed using Integrated Development Environment (IDE) i.e., Visual Studio with the help of Object-Oriented Programming Methodology in C++ programming language. The plots of various motion profiles were generated and verified using Gnuplot and MATLAB. The entire programming code has been developed and structured exclusively, taking velocity, acceleration, time, and path constraints as inputs, and generating the desired velocity profile as output. To generate various motion profiles, the final deceleration i.e., acceleration at end velocity $V_{e}$ has been considered as $0 \mathrm{~m} / \mathrm{s}^{2}$. The limits of the initial acceleration $A$ and the initial deceleration $D$ are based on the vehicle, and traffic rules which are pre-decided and taken as $5 \mathrm{~m} / \mathrm{s}^{2}$ and $-5 \mathrm{~m} / \mathrm{s}^{2}$, respectively.

\section{B. Generating various Velocity Profiles}

One of the most important aspects involved in autonomous driving is the velocity profile generation. Velocity planning has a great impact on safe driving and comfort of vehicles, especially in realistic urban circumstances. Therefore, the velocity profile must be assigned carefully on each point along the trajectory. The total length of the given trajectory (path) $S$ was divided into several minuscule paths each having a distance $s$ for which the velocity profiles were generated, considering various path constraints. The path constraints such as the maximum allowed speed $V_{m}$, the acceleration (acceleration $A$ and deceleration $D$ ), the velocity (initial velocity $V_{o}$ and end velocity $V_{e}$ ) and the total distance of the minuscule path $s$ dictated by the path were compulsorily fulfilled to obtain the desired velocity profile. Depending upon the applied constraints, the trapezoidal, linear, or ramp velocity profiles were generated. As the ramp and linear velocity profiles are components of the trapezoidal velocity profile, they help in evaluating the results for the same. The linear profile is generated in a situation when the vehicle, which is moving atits maximum velocity, decelerates to lower terminal velocity. Similarly, the ramp velocity profile is obeyed when the vehicle needs to attain an end velocity, which corresponds to its maximum speed. In addition to that, it can also trace the linear or ramp profile consisting of the only segment, $S_{1}$, or $S_{3}$ respectively, when the value of the path length is insufficient to incorporate two segments.

To verify the different types of motion profiles, velocity v/s time and velocity v/s distance (position) profiles are obtained. In the velocity v/s time profile, the velocity for every given point (time) $t$, lying on the total time taken to cover the minuscule path $T$, has been calculated. Similarly, in the velocity v/s distance profile, the velocity for every given point(distance) $p$, lying on the total distance of the minuscule paths, has been calculated. The velocity v/s time and velocity v/s distance profile comprise three segments namely, the ramp-up slope $T_{1} / S_{1}$, the constant traversal segment $T_{2} / S_{2}$, and the ramp-down slope $T_{3} / S_{3}$. On altering the values of the velocity constraints, the velocity curve decides to fit $S_{1}, S_{2}, S_{3} /$ $T_{1}, T_{2}, T_{3}$ individually, or a combination of these three segments to generate trapezoidal, linear or ramp velocity profile. Table I shows the estimated values of velocity constraints to generate different types of motion profiles. The total distance of the minuscule path $s$ for which the velocity profile has been generated is equivalent to the sum of the distance covered by three segments of the profile. The path constraint $s$ is considered as $150 \mathrm{~m}$. The distance, time, velocity, and acceleration equations required for the generation of various types of velocity profiles have been formulated using Newton's Laws of motion. The equations for $S_{1}, S_{2}$ and $S_{3}$ are necessary to calculate the distance covered by each segment in a velocity v/s distance profile. 


$$
\begin{aligned}
& V_{l}=\sqrt{V_{0}^{2}+(2 \times A \times p)} \\
& V_{2}=V_{m} \\
& \left.V_{3}=\sqrt{V_{m}^{2}+\left(2 \times D \times\left(p-\left(S_{1}+S_{2}\right)\right)\right.}\right) \\
& T_{1}=\frac{V_{m}-V_{0}}{A} \\
& T_{2}=t s_{2} \\
& T_{3}=\frac{V_{e}-V_{m}}{D} \\
& V_{l}=V_{0}+(A \times t) \\
& V_{2}=V_{m} \\
& V_{3}=V_{m}+\left(D \times\left(t-\left(T_{1}+T_{2}\right)\right)\right)
\end{aligned}
$$

\begin{tabular}{|c|c|c|c|c|c|c|c|c|}
\hline \multirow{2}{*}{ Velocity Profile } & \multicolumn{3}{|c|}{ Velocity Constraints } & \multirow{2}{*}{$\frac{\text { Time Constraint }}{T_{k} \mathrm{~S}}$} & \multicolumn{4}{|c|}{ Path Constraints } \\
\hline & $V_{0} \mathrm{~m} / \mathrm{s}$ & $V_{m} \mathbf{m} / \mathbf{s}$ & $V_{e} \mathbf{m} / \mathbf{s}$ & & $S_{1} \mathrm{~m}$ & $S_{2} \mathbf{m}$ & $S_{3} \mathbf{m}$ & Total $s \mathbf{m}$ \\
\hline Trapezoidal & 0 & 20 & 0 & 0 & 40 & 70 & 40 & 150 \\
\hline Linear & 20 & 20 & 5 & 0 & 0 & 112.5 & 37.5 & 150 \\
\hline Ramp & 0 & 20 & 20 & 0 & 40 & 110 & 0 & 150 \\
\hline Trapezoidal (no $S_{2}$ Region) & 0 & 20 & 0 & 0 & 40 & 0 & 40 & 80 \\
\hline Trapezoidal (new $\left.V_{\text {stable }}\right)$ & 0 & 20 & 0 & 6 & 10 & 60 & 10 & 80 \\
\hline
\end{tabular}

\begin{tabular}{|c|c|c|c|c|c|c|c|c|}
\hline \multirow{2}{*}{ Velocity Profile } & \multicolumn{4}{|c|}{ Non-Smooth Curve } & \multicolumn{4}{|c|}{ Smooth s-Curve } \\
\hline & $T_{1} \mathrm{~S}$ & $T_{2} \mathrm{~S}$ & $T_{3} \mathrm{~S}$ & Total $T \mathrm{~s}$ & $T_{1} \mathrm{~S}$ & $T_{2} \mathbf{S}$ & $T_{3} \mathrm{~S}$ & Total $T$ s \\
\hline Trapezoidal & 4 & 3.5 & 4 & 11.5 & 3.745 & 3.5 & 3.745 & 10.99 \\
\hline Linear & 0 & 5.625 & 3 & 8.625 & 0 & 5.625 & 3 & 8.625 \\
\hline Ramp & 4 & 5.5 & 0 & 9.5 & 3.49 & 5.5 & 0 & 8.99 \\
\hline Trapezoidal (no $S_{2}$ Region) & 4 & 0 & 4 & 8 & 3.745 & 0 & 3.745 & 7.49 \\
\hline Trapezoidal (new $\left.V_{\text {stable }}\right)$ & 2 & 6 & 2 & 10 & 1.87 & 6 & 1.87 & 9.74 \\
\hline
\end{tabular}

Table 1: Velocity, Time and Distance Constraints Considered for Generating Various Types of Velocity Profiles

Table 2: Time Taken to Cover Each Segment of Various Non-Smooth and Smooth S-curve Velocity Profile

Using Equations 1, 2 and 3, the distance covered by each segment of the velocity v/s distance profile can be derived. The time $t_{S 2}$, required to cover the segment $S_{2}$, is obtained using Equation 5. The velocity at any given point (distance) $p$ lying between 0 to $S_{1}, S_{1}$ to $S_{2}$, and $S_{2}$ to $S_{3}$ on the minuscule path in a velocity v/s distance profile, can be calculated using Equations 16, 17 and 18 respectively. Table I shows the distance covered by each segment of the various generated velocity v/s distance motion profiles to the considered velocity, acceleration, path, and time constraints.

Newton's Laws of motion can be also used to formulate the equations in the simplest form of acceleration, velocity, and time constraints for $T_{1}, T_{2}$, and $T_{3}$, to calculate the time taken by each segment of the velocity v/s time profile. Using Equations 19, 20 and 21, the time taken to cover each segment of the velocity v/s time profile can be obtained. The total time to travel the minuscule path $s$ is equal to the sum of the time taken by each individual segment of the profile. The velocity at any given point (time) $t$ lying between 0 to $T_{1}, T_{1}$ to $T_{2}$ and $T_{2}$ to $T_{3}$ on various velocity curves in a velocity v/s 
time profile, can be calculated using Equations 22, 23 and 24 respectively. Table II presents the time taken by each segment of the various generated smooth and non- smooth velocity v/s time profiles to the considered velocity, acceleration, path, and time constraints.

The non-smooth trapezoidal, linear and ramp velocity profiles w.r.t distance and time are generated in Figures 5, 6 and 7, respectively. The velocity, acceleration, path, and time constraints have been applied to obtain each profile. To maintain constant velocity as the leading vehicle, the acceleration at endvelo city $V_{e}$ in all the profiles is considered to be $0 \mathrm{~m} / \mathrm{s}^{2}$. The trapezoidal curve comprises of all the three segments i.e., $S_{1}, S_{2}$ and $S_{3}$ in velocity v/s distance profile, and $T_{1}, T_{2}$ and $T_{3}$ in velocity v/s time profile. The linear velocity curve does notconta in $S_{1}$ and $T_{1}$ segments, whereas the ramp velocity curve does not contain $S_{3}$ and $T_{3}$ segments in the respective distance and time profiles. Also, the total time taken to cover the entire profile for a given length of minuscule path is calculated and tabulated in Table 2.

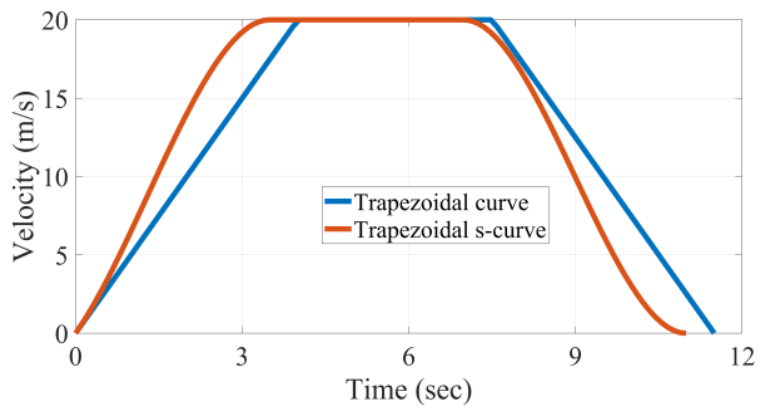

Velocity v/s Time

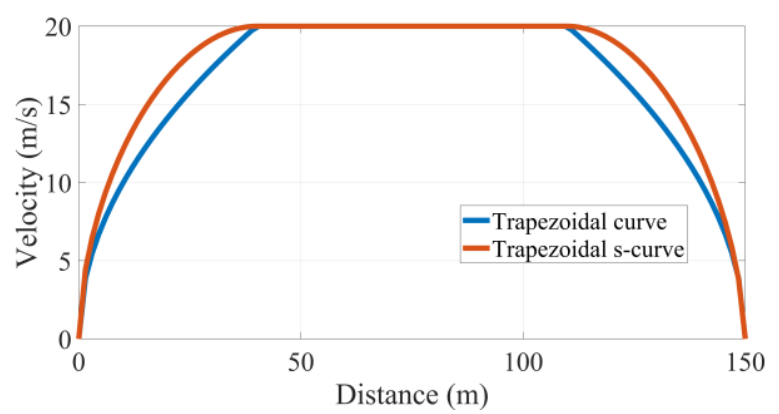

Velocity v/s Distance

Figure 5: Non-Smooth and Smooth s-Curve Trapezoidal Velocity Profile

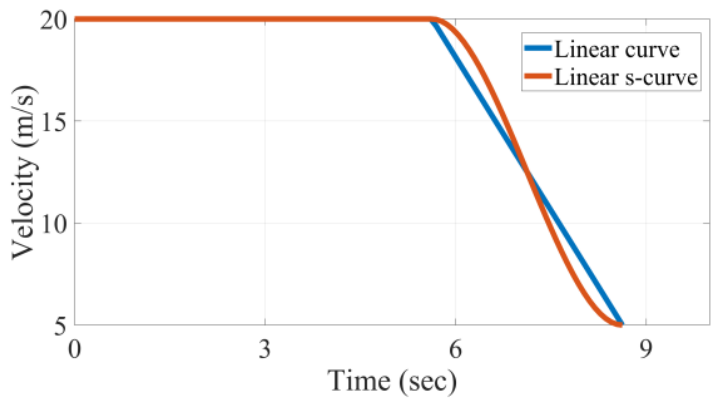

Velocity v/s Time 


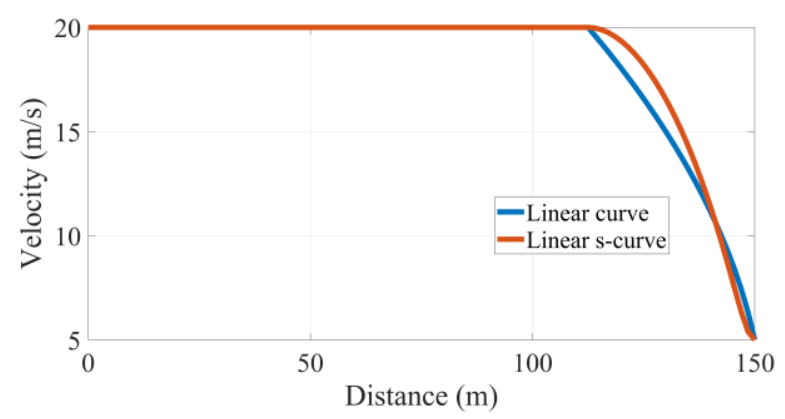

Velocity v/s Distance

Figure 6: Non-Smooth and Smooth S-Curve Linear Velocity Profile

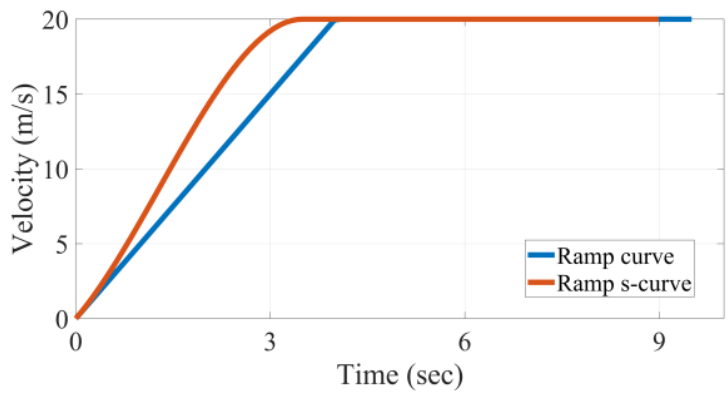

Velocity v/s Time

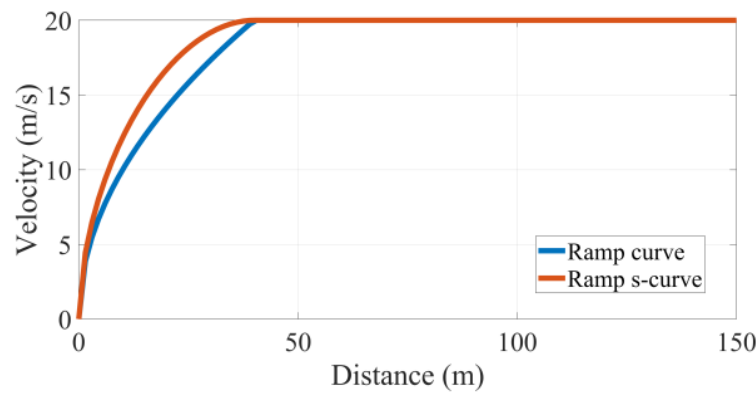

Velocity v/s Distance

Figure 7: Non-Smooth and Smooth s-Curve Ramp Velocity Profile.

The above section deals with the plotting of a non-smooth velocity profile curve within the applied constraints and aims at generating a curve that requires the least amount of time to travel from the initial to the final position on the trajectory. Amongst the three types of curves obtained, the trapezoidal velocity curve is the most applicable as it guarantees the employment of the segment $S_{2}$ which maintains the vehicle at constant maximum velocity. The ability of jerk avoidance of the system is furnished by $T_{k}$, which alters the maximum velocity to a lower value for maintaining stability. This ensures that the vehicle travels at the maximum velocity for the longest time possible hence reducing the duration of travel to a minimum.

\section{To Smoothen the Generated Velocity Profiles}

Following the generation of various velocity profiles is the curve smoothening, which is necessary to provide smooth control and jerk minimization. The time duration required to cover the ramp-up and ramp-down segments in the s-curve profile differ from those obtained in the trapezoidal curve profile. 


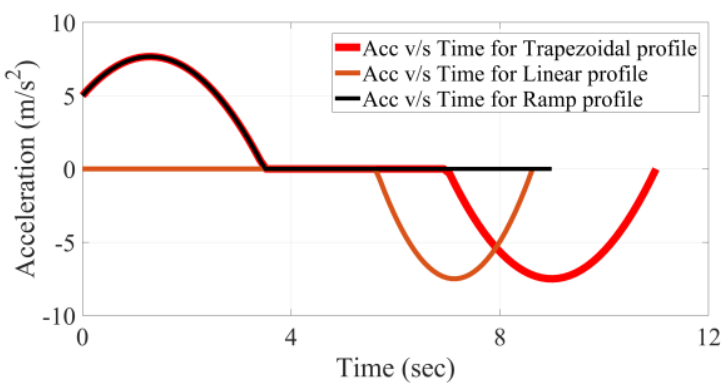

Figure 8: Acceleration v/s Time Curve for Various S-Curve Profiles.

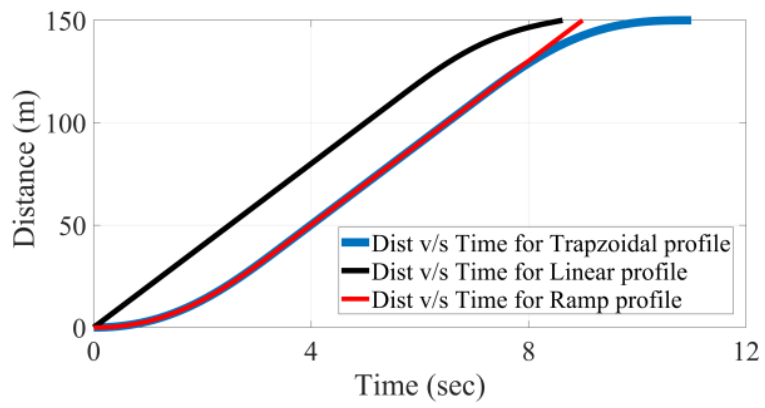

Figure 9: Distance v/s Time Curve for Various S-Curve Profiles.

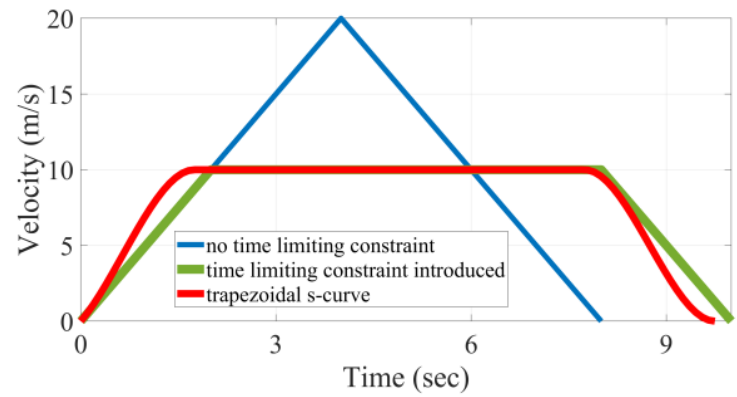

Velocity v/s Time

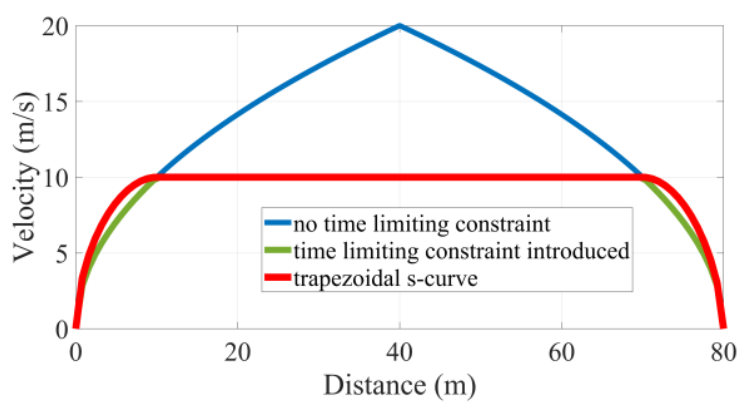

Velocity v/s Distance

Figure 10: Generation of a Trapezoidal Velocity Profile using $n e w V_{m}$ on Introduction of a

Time Limiting Constant, $T_{k}$.

The total time $t_{f}$, required to cover each segment in an s-curve profile, is calculated to produce a continuous scurve. In this approach, the ramp-up and ramp-down segments are smoothened individually using cubic polynomials for all values of $t$ ranging from $0-t_{f}$ in the velocity v/s time s-curve profile. Similarly, the velocity v/s distance s-curve is generated for all values of $p$ from $0-s_{f}$. The generated non-smooth trapezoidal, linear, and ramp velocity profile in Figures 
5, 6 and 7 respectively, are adopted to smoothen the curve using spline equations considering the same velocity, acceleration, path, and time constraints. Table II shows the variation in travel time for generated non-smooth profiles as opposed to the s-curve velocity profiles. Moreover, the value of $t_{f}$ is used to obtain acceleration and distance s-curve profiles. In addition to velocity, acceleration and distance are also calculated for all values of $t$ from $0-t_{f}$ in each segment of the s-curve motion profile, thus obtaining the smoothened acceleration v/s time and distance v/s time profiles. Figures 8 and 9 display the acceleration $\mathrm{v} / \mathrm{s}$ time and distance $\mathrm{v} / \mathrm{s}$ time s-curve profile for trapezoidal, linear, and ramp s-curve motion profiles, respectively.

In case the time required to travel segment $S_{2}$ i.e., $t_{S 2}$ turns out to be lesser than the time limiting constraint $T_{k}$, a new value of maximum velocity $V_{m}$ is calculated, considering the given velocity, acceleration, time, and path constraints. Figure 10 demonstrates the purpose of the time limiting constraint, $T_{k}$. The simulation of the profiles considers the path constraint, i.e., the total length of the minuscule path $s$, to be $80 \mathrm{~m}$. The velocity and time constraints applied for the generation of the profiles are discussed in Table I. The profile generated observing the time defining constraint, $T_{k}$, equal to 0 seconds, is extremely unstable due to an abrupt change from an accelerating to a decelerating region, resulting in a jerky motion. A trapezoidal velocity profile is implemented to avoid the jerk. The trapezoidal curve is fitted for the given distance by calculating the new maximum velocity $n e w V_{m}$, lesser than the original, considering the time limiting constant, $T_{k}$. When the value of $T_{k}$ is considered to be 6 seconds, the value of $n e w V_{m}$ is estimated to be $10 \mathrm{~m} / \mathrm{s}$ for generating the trapezoidal profile. The trapezoidal curve is then smoothened using polynomial splines to generate the respective s-curve. The distance traveled, and the time taken by every individual segment, viewing the time limiting constraint $T_{k}$, in the generated non-smooth and smooth s-curve profiles are tabulated in Tables I and II, respectively.

In addition to this, a few exceptional cases were also reviewed during the plotting of various velocity profiles. A distinct case where the initial velocity was assumed to be $20 \mathrm{~m} / \mathrm{s}$, the maximum velocity as $10 \mathrm{~m} / \mathrm{s}$, and the end velocity as $15 \mathrm{~m} / \mathrm{s}$, traces a profile as shown in Figure 11. The values of acceleration and deceleration are considered as $5 \mathrm{~m} / \mathrm{s}^{2}$ and $5 \mathrm{~m} / \mathrm{s}^{2}$ respectively. According to the vehicular constraints, the value of maximum velocity cannot exceed the end velocity and thus, the graph does not trace an accelerating section. Such a situation can be encountered when the vehicle, which is currently travelling at a higher velocity comes across a steep curvature which constrains the vehicle to adhere to a lower value of the maximum velocity.

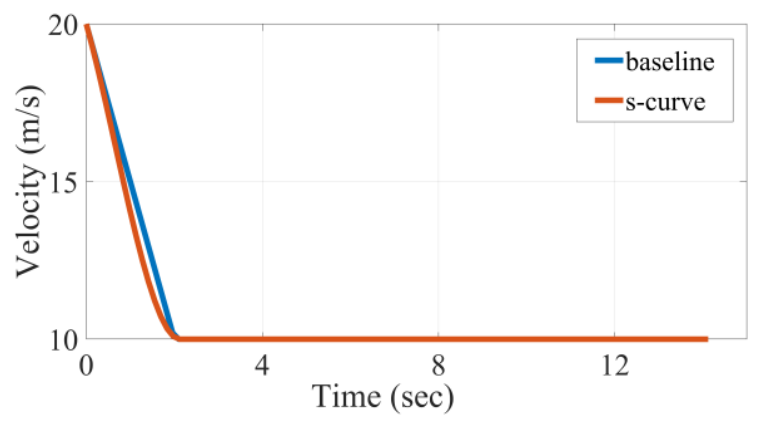

Velocity v/s Time 


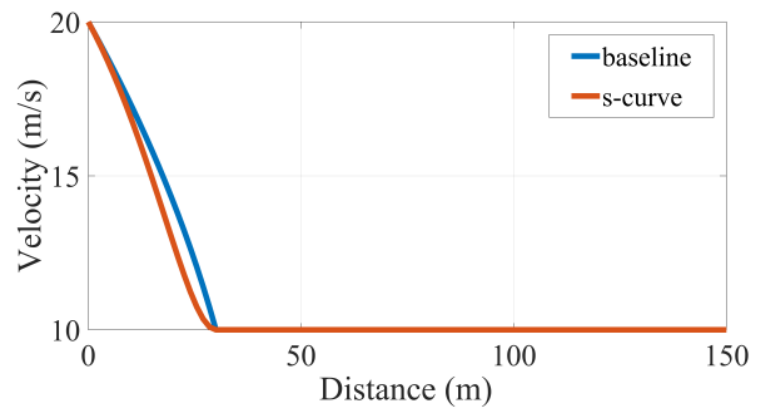

Velocity v/s Distance

Figure 11: Generation of a Velocity Profile with the Absence of an Accelerating Segment when $V_{e}$ is Greater than $V_{m}$.

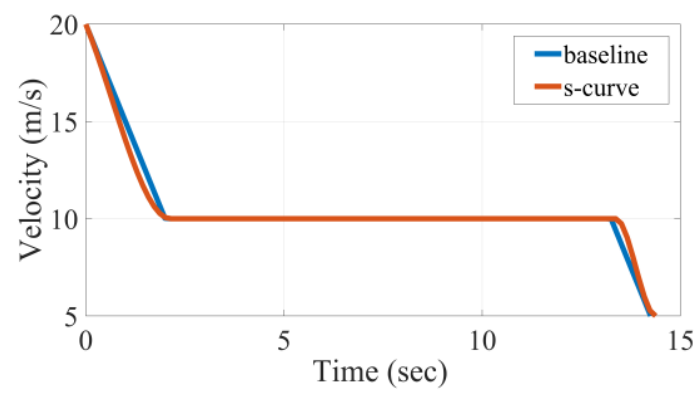

Velocity v/s Time

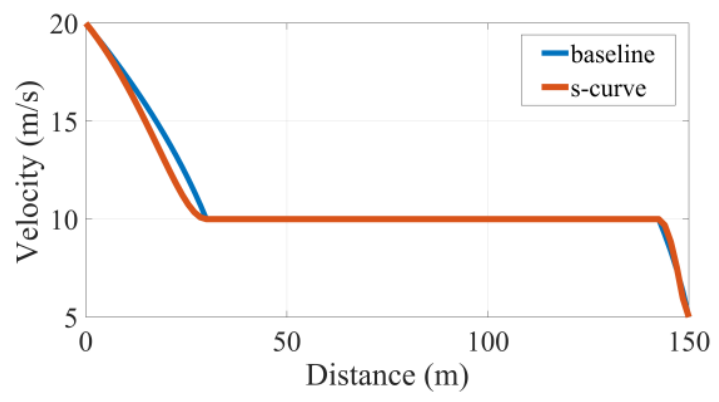

Velocity v/s Distance

Figure 12: Generation of a Velocity Profile with an Additional Decelerating Segment when $V_{e}$ is Lesser than $V_{m}$.

Likewise, when the values of the initial, maximum and end velocity are $20 \mathrm{~m} / \mathrm{s}, 10 \mathrm{~m} / \mathrm{sand} 5 \mathrm{~m} / \mathrm{s}$ respectively, the velocity profile traces an additional decelerating section, as presented in Figure 12, as no constraints are overridden in this particular case.

The generation of an acceleration continuous s-profile curve contributes in minimizing the jerk to a minimum value by avoiding abrupt changes in the values of velocity and acceleration during the plotting of the trapezoidal velocity curve. The final acceleration is assumed to be $0 \mathrm{~m} / \mathrm{s}^{2}$ ensures that the vehicle maintains a constant final velocity $V_{e}$. The time duration necessary for travelling equal distances in the smooth and non-smooth profiles turns out to be dissimilar for the trapezoidal and ramp profile curves. The varying time is the outcome of the application of a steeper slope such as in $S_{l}$ region for the generation of s-profile curve as opposed to the non-smooth profile. Consequently, the interpolation of cubic spline curve is suitable for the velocity profile algorithm as it acquires an edge over the non-smooth curve both in terms of minimum time and improved stability. 


\section{CONCLUSIONS}

Autonomous vehicles have been extensively studied over the past decades to meet a variety of dynamic, kinematic, and environmental constraints. The two major aspects of autonomous driving include path planning and the velocity planning algorithm. This paper critically reviewed existing velocity planning approaches applied to autonomous systems and developed a method to produce an optimal or near-optimal solution to the problem with low computational cost. Velocity profile generation, an effective tool for motion control is achieved by plotting a curve for the minimum time travel of the vehicle through the specified path within given acceleration limits. The velocity profile ensures the safety, comfort, and efficiency of the system and deals with the real-time implementation for autonomous vehicles. The production of comfort constrained profiles for acceleration and velocity analyzes the behavior and ability of the system to generate minimum absolute jerk.

Considering the various constraints applied to the system, the best fitting curve for velocity and acceleration is obtained while ensuring a smooth and non-jerky motion. An integration of this concept is presented which yields successful results in the experiments. However, it is difficult to produce a near ideal system and this approach bears its limitations as well. The quadratic formula dealing with the calculation of the new value of maximum velocity, new $V_{m}$, fails to obtain a suitable solution if no root is found. In that case, the constraints are noto beyed as a whole and need a way to be dealt with. In addition to this, the smoothening parameters have to be evaluated separately which increases the computational speed slightly. The strategy is conservative, and experiments have to be doneat various stages with a wide range of inputs for perfect incorporation and implementation of this method, to realistic urban driving scenarios. The results obtained in this paper motivate continued work in interpolating the velocity profile generation in the path planning and trajectory generation algorithm. Itcan also be tested on a broader range of realistic urban traffic scenarios driving at higher speeds to verify and evaluate its performance more sufficiently. The proposed method promises to interact and fit well with the existing systems and evolve with the entire autonomous driving system.

\section{REFERENCES}

1. K. Chu, M. Lee, and M. Sunwoo, "Local path planning for offroadautonomous driving with avoidance of static obstacles," IEEETransactions on Intelligent Transportation Systems, vol. 13, no. 4, pp.1599-1616, 2012

2. Avantsa V. S. S. Somasundar, G. Yedukondalu\& K. Shiva Kesavulu, "Singularity Analysis of Kuka 6 DOF Robot for Motion Simulation”, International Journal of Mechanical and Production Engineering Research and Development (IJMPERD), Vol. 9, Issue 2, pp, 223-228

3. C. L. Bianco, A. Piazzi, and M. Romano, "Velocity planning forautonomous vehicles," in IEEE Intelligent Vehicles Symposium, 2004.IEEE, 2004, pp. 413-418.

4. SupriyaSahu\& B. B Choudhury, "Cycle Time Optimized Path Planning for Industrial Robot using Robomaster", International Journal of Mechanical and Production Engineering Research and Development (IJMPERD) ,Vol. 7, Issue 5, pp, 95-102

5. S. Thrun, "Toward robotic cars," Communications of the ACM, vol. 53,no. 4, pp. 99-106, 2010.

6. G. D. Awchat\& Yamini. N. Deshmukh, "Seismic Response of Tall Building with Underground Storey using Dampers", International Journal of Civil Engineering (IJCE) ,Vol. 6, Issue 4, pp; 57-66

7. L. D. Burns, “A vision of our transport future,” Nature, vol. 497, no.7448, pp. 181-182, 2013. 
8. J. Kim, K. Jo, D. Kim, K. Chu, and M. Sunwoo, "Behavior and pathplanning algorithm of autonomous vehicle al in structured environments, "IFAC Proceedings Volumes, vol. 46, no. 10, pp. 36-41, 2013.

9. K. Rebecca Jebaseeli Edna, V. Jemmy Joyce \& S. Deva Arul, "Control Limits for Variable Fraction Defective - P Chart”, International Journal of Applied Mathematics \& Statistical Sciences (IJAMSS) ,Vol. 3, Issue 2, pp, 29-32

10. V. T. Minh and J. Pumwa, "Feasible path planning for autonomousvehicles," Mathematical Problems in Engineering, vol. $2014,2014$.

11. J. Villagra, V. Milan'es, J. P. Rastelli, J. Godoy, and E. Onieva, "Path andspeed planning for smooth autonomous navigation," in IV 2012 - IEEEIntelligent Vehicles Symposium, Jun 2012, Alcala de Henares, Madrid,Spain, 2012.

12. L. Claussmann, M. Revilloud, D. Gruyer, and S. Glaser, "A review ofmotion planning for highway autonomous driving," IEEE Transactionson Intelligent Transportation Systems, vol. 21, no. 5, pp. 1826-1848,2019.

13. F. Altch'e, P. Polack, and A. de La Fortelle, "High-speed trajectoryplanning for autonomous vehicles using a simple dynamic model," in2017 IEEE 20th International Conference on Intelligent TransportationSystems (ITSC). IEEE, 2017, pp. 1-7.

14. L. Biagiotti and C. Melchiorri, Trajectory planning for automaticmachines and robots. Springer Science \& Business Media, 2008 .

15. C. Katrakazas, M. Quddus, W.-H. Chen, and L. Deka, "Real-time motionplanning methods for autonomous on-road driving: State-of-the-art andfuture research directions," Transportation Research Part C: EmergingTechnologies, vol. 60, pp. 416$442,2015$.

16. T. Gu and J. M. Dolan, "On-road motion planning for autonomousvehicles," in International Conference on Intelligent Robotics and Applications.Springer, 2012, pp. 588-597.

17. J. P. Talamino and A. Sanfeliu, "Anticipatory kinodynamic motion plannerfor computing the best path and velocity trajectory in autonomousdriving," Robotics and autonomous systems, vol. 114, pp. 93-105, 2019.

18. Y. Kanayama, Y. Kimura, F. Miyazaki, and T. Noguchi, "A stable trackingcontrol method for an autonomous mobile robot," in Proceedings.,IEEE International Conference on Robotics and Automation. IEEE,1990, pp. 384-389.

19. R. Solea and U. Nunes, "Trajectory planning with velocity planner forfully-automated passenger vehicles," in 2006 IEEE Intelligent TransportationSystems Conference. IEEE, 2006, pp. 474-480.

20. X. Li, Z. Sun, D. Cao, Z. He, and Q. Zhu, "Real-time trajectoryplanning for autonomous urban driving: Framework, algorithms, andverifications, "IEEE/ASME Transactions on mechatronics, vol. 21, no. 2,pp. 740-753, 2015.

21. E. Velenis and P. Tsiotras, "Optimal velocity profile generation for givenacceleration limits: Receding horizon implementation," 072005 , pp.2147 - 2152 vol. 3.

22. I. Bae, J. Moon, J. Jhung, H. Suk, T. Kim, H. Park, J. Cha, J. Kim,Kim, and S. Kim, "Self-driving like a human driver instead of arobocar: Personalized comfortable driving experience for autonomousvehicles, " arXiv preprint arXiv:2001.03908, 2020.

23. J. Najmi and S. I. A. Shah, "Analysis of velocity profile for laminarflow in a round pipe."

24. A. Rahatgaonkar, "Velocity planning approach for autonomous vehicles, "Master's thesis, 2016.

25. W. Xu, J. Pan, J. Wei, and J. M. Dolan, “Motion planning under uncertaintyfor on-road autonomous driving," in 2014 IEEE InternationalConference on Robotics and Automation (ICRA). IEEE, 2014, pp.2507-2512.

26. S. Lefevre, A. Carvalho, and F. Borrelli, “A learning-based frameworkfor velocity control in autonomous driving," IEEE Transactions onAutomation Science and Engineering, vol. 13, no. 1, pp. 32-42, 2015. 
27. Y.-R. G. Zvi Shiller, “Dynamic motion planning of autonomous vehices, ”IEEE Transactions on Robotics and Automation, vol. 7, pp. 241-249,1991.

28. M. Adams and J. Ibanez-Guzman, "Limiting velocity \& accelerationcommands for dynamic control of a large vehicle," in 7th InternationalConference on Control, Automation, Robotics and Vision, 2002. ICARCV2002., vol. 3. IEEE, 2002, pp. 14751480.

29. J. Y. Seiji Sugiyama and T. Yoshikawa, "Path planning of a mobilerobot for avoiding moving obstacles with improved velocity control byusing the hydrodynamic potential," The 2010 IEEE/RSJ InternationalConference on Intelligent Robots and Systems, pp. 1421-1426, 2010.

30. C. Chen, Y. He, C. Bu, J. Han, and X. Zhang, "Quartic b'eziercurve-based trajectory generation for autonomous vehicles with curvatureand velocity constraints, " in 2014 IEEE International Conference onRobotics and Automation (ICRA). IEEE, 2014, pp. 6108-6113.

31. L. Consolini, M. Locatelli, A. Minari, and A. Piazzi, "A linear-timealgorithm for minimum-time velocity planning of autonomous vehicles, "in 2016 24th Mediterranean Conference on Control and Automation(MED). IEEE, 2016, pp. 490-495. 